\title{
ANALYSIS OF SELF - GRAVITATING PLANETARY SATELLITES IN THE SOLAR SYSTEM
}

\author{
S.O.Yasenev \\ Dpt of Aerospace Surveying, National Aviation University \\ Kyiv, Ukraine,yasenev91@gmail.com
}

\begin{abstract}
As of today there have been more than 180 planetary satellites discovered in the Solar system, and the number of outer moons found continues to grow. Most of those natural satellites have insufficient mass and are able to retain their shape only due to the strength of the electromagnetic force. The purpose of this paper is to analyze the moons' physical properties. The analysis of planetary satellites as self - gravitating bodies, i.e. celestial bodies which rely on the weight of their own mass and resulting gravitational force to maintain their shape and tend to bring it closer to the hydrostatic equilibrium, was performed.
\end{abstract}

Key words: planetary satellites; gravitational field; shape; mass; classification.

\section{Introduction}

Modern astronomical observations carried out by space - and ground - based means resulted in the discovery of a large number of planetary satellites - more than 180, and this number continues to grow. But most of these moons have small masses while their shape and size are kept fixed by the strength of electromagnetic interactions in their composite minerals. Due to that all small planetary satellites are irregular in shape $[2,5]$.

On the other side, the planets in the Solar System also have massive moons that keep their spherical shape by their own gravity; in other words, the gravitational field and proper rotation had a paramount importance during their evolution. It is evident that those moons significantly differ from smaller ones, most of which have been discovered over the last twenty years (Fig. 1).

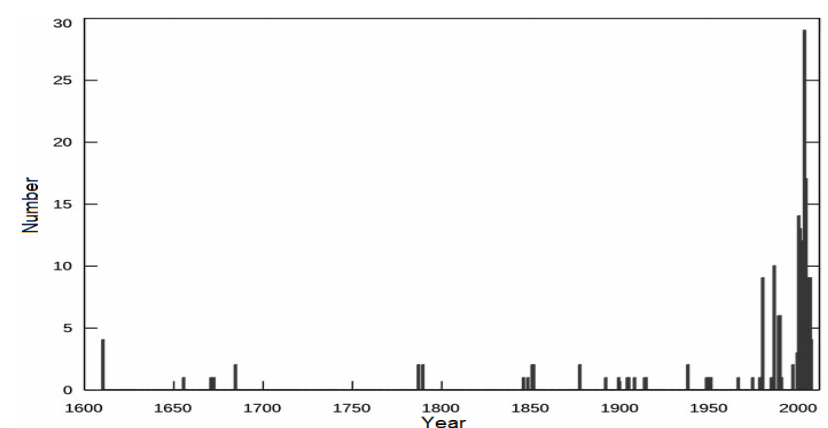

Figure 1: Number of planetary satellites discovered over the course of a year.
In view of the above, the problem of classification of planetary satellites in the Solar System by their size, distribution of mass, gravitational field, shape, etc. has arisen.

The problem of investigation of planets and their moons by spacecraft means is important and requires the most thorough study of their characteristics by methods of celestial mechanics.

\section{Problem statement}

This study is focused on planetary satellites, the so called self - gravitating bodies, i.e. bodies which retain their shape by their own mass, mass distribution and resulting gravitational force and tend to bring it closer to the equilibrium. Such moons are called either planets or planetoids or true companions.

What are the criteria to distinguish such planetoids among a total of over 180 planetary satellites? We selected the total mass of a moon as the first criterion while its size is the second criterion and its shape is the third one. It is obvious that a planetary satellite should have a shape close to spherical to be assigned as a planetoid.

The objective of this study is to establish the mentioned criteria and detect the planetary satellites in the Solar System which meet those criteria.

By now there have been a series of studies of this subject carried out by numerous scientists, including K.Kholshenikov, L.Sokolov, B.Kondratiev, O.Zheleznyak, A.Zavizion, A.Vidmachenko, A.Morozhenko, L.Konstantinovskaya, E.Ruskol, A.Ivanov, V.Uralskaya and others.

\section{Materials of the study}

It was found that the largest moons have a radius which is within the range from 0.002 to 0.270 of the host planet radius. The only exception is Pluto and its largest moon - Charon. For most of the planets (except for the Earth and Pluto), this range is rather narrow - from 0.002 to 0.042 of the host - planet radius [3].

All major moons (except Triton) are synchronized with their host planets; it means that such a moon rotates with the same side facing its host planet orbiting the Sun (Table 1).

Planetary satellites deviate from hydrostatic equilibrium; hence, they tend to have a shape different from a 
spheroid and asymmetric with respect to the axis of rotation and equatorial plane. Besides, unlike asteroids, large moons are evolving objects, so they have inner cores, magnetic fields (some of them), and regular shape [4, 6].

Table 1. Physical characteristics of different moons

\begin{tabular}{|c|l|c|c|c|c|}
\hline № & $\begin{array}{c}\text { Name } \\
\text { of the } \\
\text { moon }\end{array}$ & $\begin{array}{c}\text { Mass, } \\
\mathbf{1 0}^{\mathbf{2 0}}\end{array}$ & $\begin{array}{c}\text { Density, } \\
\mathbf{k g} \cdot \mathbf{m}^{-\mathbf{3}}\end{array}$ & $\begin{array}{c}\text { Mean } \\
\text { radius, } \\
\mathbf{k m}\end{array}$ & $\begin{array}{c}\text { Host } \\
\text { planets }\end{array}$ \\
\hline 1 & Ganymede & 1481.90 & 1936 & 2634.10 & Jupiter \\
\hline 2 & Titan & 1345.20 & 1880 & 2576.00 & Saturn \\
\hline 3 & Callisto & 1077.00 & 1835 & 2410.30 & Jupiter \\
\hline 4 & Io & 893.19 & 3528 & 1821.00 & Jupiter \\
\hline 5 & Moon & 734.80 & 3347 & 1737.10 & Earth \\
\hline 6 & Europe & 480.20 & 3019 & 1561.00 & Jupiter \\
\hline 7 & Triton & 214.00 & 2061 & 1354.00 & Neptune \\
\hline 8 & Titania & 35.27 & 1711 & 788.50 & Uranus \\
\hline 9 & Oberon & 30.14 & 1630 & 761.50 & Uranus \\
\hline 10 & Rhea & 23.07 & 1234 & 764.50 & Saturn \\
\hline 11 & Iapetus & 18.06 & 1089 & 735.80 & Saturn \\
\hline 12 & Charon & 15.80 & 1650 & 606.00 & Pluto \\
\hline 13 & Ariel & 13.50 & 1670 & 578.90 & Uranus \\
\hline 14 & Umbriel & 11.75 & 1405 & 584.30 & Uranus \\
\hline 15 & Dione & 10.96 & 1479 & 562.00 & Saturn \\
\hline 16 & Tethys & 6.18 & 0986 & 531.20 & Saturn \\
\hline 17 & Enceladus & 1.10 & 1610 & 252.20 & Saturn \\
\hline 18 & Miranda & 0.71 & 1214 & 236.50 & Uranus \\
\hline 19 & Proteus & 0.44 & 1300 & 210.00 & Neptune \\
\hline 20 & Mimas & 0.38 & 1160 & 198.60 & Saturn \\
\hline 21 & Nereid & 0.31 & 1150 & 170.00 & Neptune \\
\hline 22 & Phoebe & 0.08 & 1638 & 106.50 & Saturn \\
\hline & & & & & \\
\hline
\end{tabular}

Let us conduct an analysis of mass and size of a celestial body that can have any shape and does not tend to bring it closer to theequilibrium. Suppose the body's mass equals $m$, its density is $\rho$; then, its volume is $V=\frac{m}{\rho}$, thus, the radius is $R=\left(\frac{m}{\rho}\right)^{\frac{1}{3}}$.

Given that a celestial body is a sphere, we can define the acceleration of gravity on its surface by the formula:

$$
g=\frac{G m}{R^{2}}=G m^{\frac{1}{3}} \rho^{\frac{2}{3}}
$$

where $G$ is the gravitational constant.

Now we can estimate the pressure created at the centre of the celestial body by gravity. The pressure created by an uniform column with the height of $R$ in the gravitational field $g$ is defined as follows: $P=\rho g R$.

Then, the pressure inside the celestial body equals to:

$$
P=\rho g R=G m^{\frac{2}{3}} \rho^{\frac{4}{3}}
$$

Thus, there is the pressure on the body from all directions, and if the body shape is unable to withstand the effect of that force, in other words, the body is not spherical, the shift (deformation) resulted from the shear stress occurs. Since elastic solids are able to withstand the shear stress to some extent, celestial bodies of arbitrary shape are likely to exist.

If the shape of a celestial body is different from a sphere, the shear stress $\sigma$ in it is of the same order as the pressure $P$ :

$$
\sigma=P=G m^{\frac{2}{3}} \rho^{\frac{4}{3}}
$$

Solid bodies are able to resilient response to the shear stress, but every substance has limited ability to resist. In other words, every solid celestial body has its tensile strength - suppose it equals $\sigma_{m}$. Under the shear stresses that are greater than $\sigma_{m}$, a solid celestial body undergoes irreversible changes in its shape.

Now, based on the conditions $\sigma=\sigma_{m}$, we can estimate the critical mass $m_{\kappa p}$ and critical radius $R_{\kappa p}$ with which the celestial bodies can only have a shape close to spherical [1].

We can define the critical mass by the formula:

$$
m_{\kappa p}=\frac{1}{\rho^{2}}\left(\frac{\sigma_{m}}{G}\right)^{\frac{3}{2}}
$$

We can determine the critical radius by the formula:

$$
R_{\kappa p}=\frac{1}{\rho^{2}}\left(\frac{\sigma_{m}}{G}\right)^{\frac{1}{2}}
$$

Taking into account that most planetary satellites in the Solar system with the shape close to spherical are icy moons, we can suppose that the lower limit to the density is $\rho=1000 \mathrm{~kg} / \mathrm{m}^{3}$ while the tensile strength of ice is $\sigma_{m}=3 \cdot 10^{6} \mathrm{~N} / \mathrm{m}^{2}$ (Table 2).

It is obvious that whether satellite reaches equilibrium shape (i.e. approaches to the spherical shape) or not depends on its composite material (Fig. $2-3$ ).

It is clear from the figures that a planetoid or a self gravitating body in the Solar system, which retains its shape by the weight of its own mass, mass distribution and resulting gravitational force and tends to bring it closer to the equilibrium, is a planetary satellite with the mass and mean radius exceeding critical ones. The smallest moon in the Solar system which meets the above requirements is Miranda.

Table 2. Critical masses and radii for celestial bodies composed of different materials with different densities

\begin{tabular}{|l|c|c|c|c|}
\hline \multirow{2}{*}{ Parameters } & \multicolumn{4}{|c|}{ Composite materials } \\
\cline { 2 - 5 } & Ice & Regolith & Granite & Iron \\
\hline $\begin{array}{l}\text { Density, } \\
\mathrm{kg} / \mathrm{m}^{3}\end{array}$ & 1000 & 2500 & 2700 & 7800 \\
\hline $\begin{array}{l}\text { Tensile strength, } \\
\mathrm{N} / \mathrm{m}^{2}\end{array}$ & $3 \cdot 10^{6}$ & $3 \cdot 10^{7}$ & $1 \cdot 10^{8}$ & $1 \cdot 10^{9}$ \\
\hline $\begin{array}{l}\text { Critical mass, } \\
\mathrm{kg}\end{array}$ & $1 \cdot 10^{19}$ & $3 \cdot 10^{19}$ & $3 \cdot 10^{20}$ & $1 \cdot 10^{21}$ \\
\hline $\begin{array}{l}\text { Critical radius, } \\
\mathrm{km}\end{array}$ & 212 & 268 & 453 & 496 \\
\hline
\end{tabular}




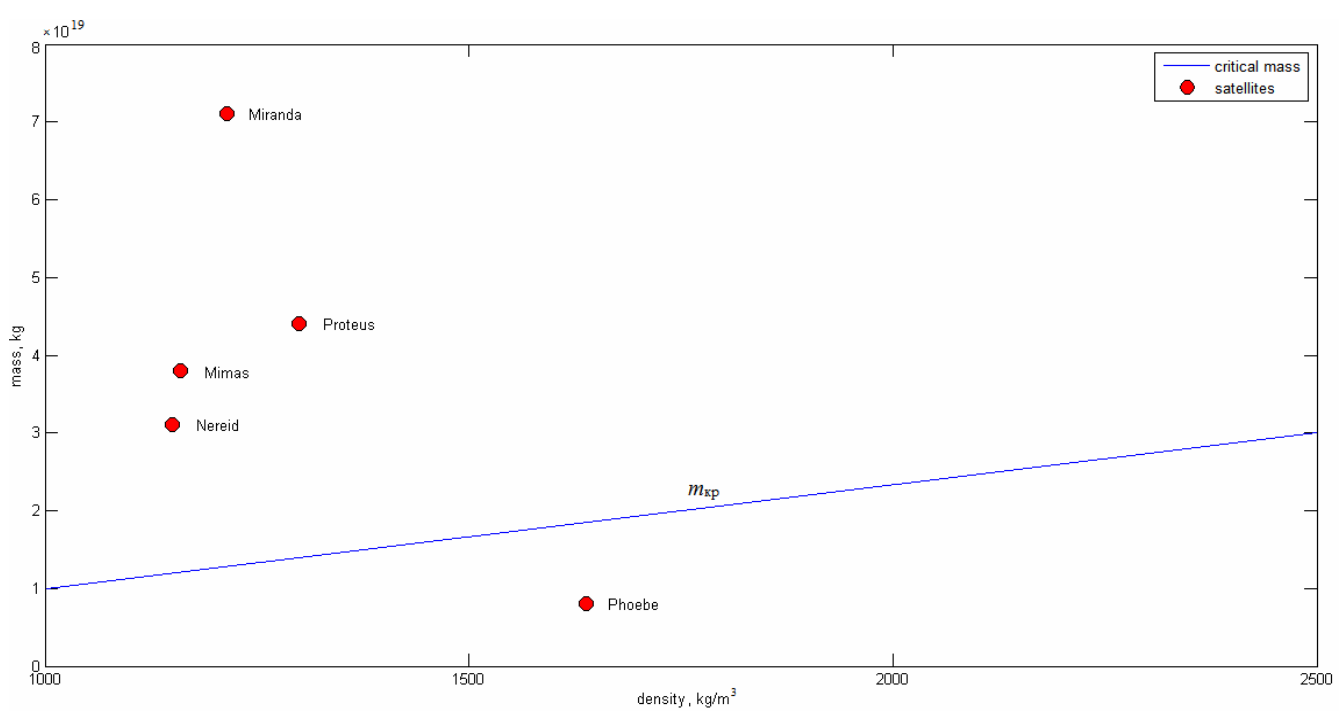

Figure 2: Critical masses for different moons.

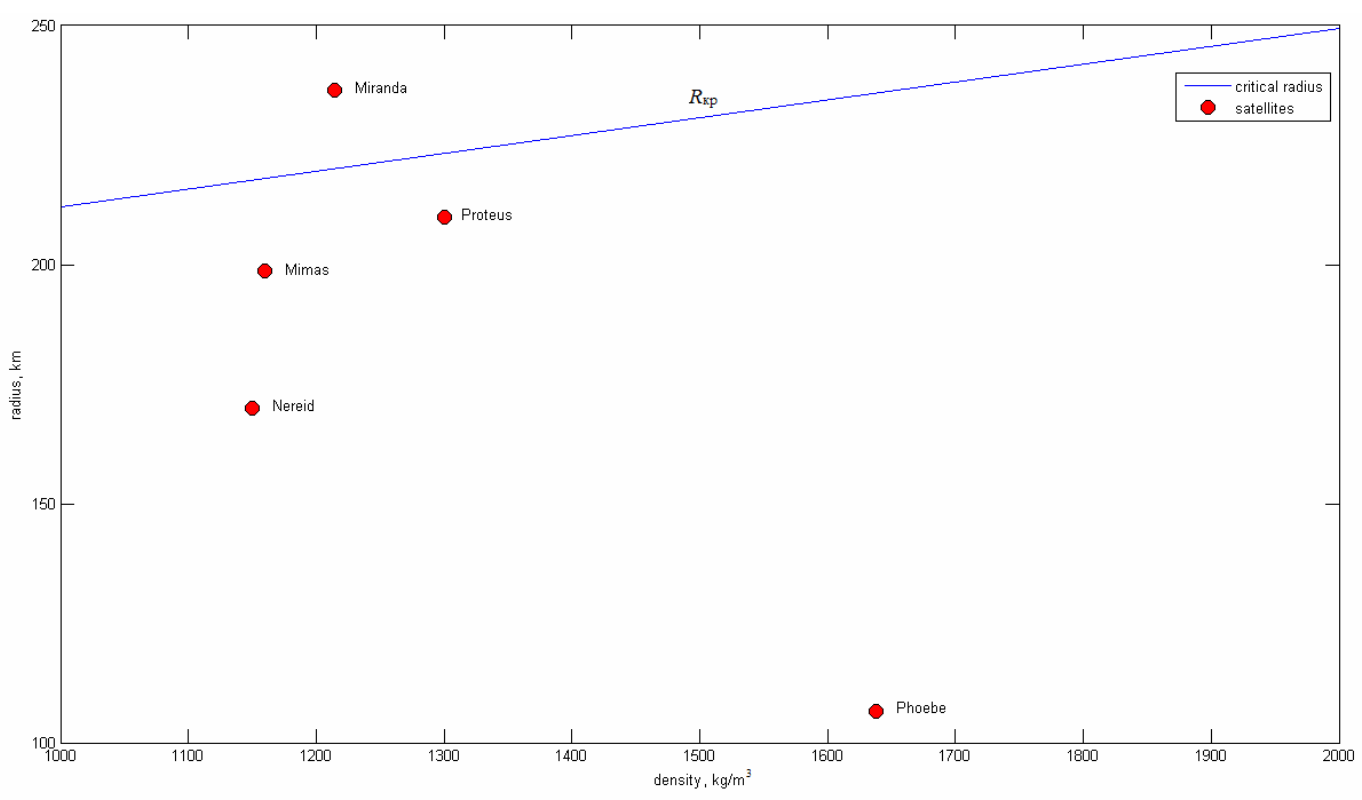

Figure 3: Critical radii for different moons.

\section{Conclusions}

It has been found that a natural planetary satellite can be referred to as a planetoid if it is a celestial body of spherical shape with the mass of over $10^{19} \mathrm{~kg}$ and mean radius of over $212 \mathrm{~km}$. Celestial bodies with smaller masses remain firm and retain their irregular shapes.

According to the afore - indicated criteria, only 18 moons can be selected from over 180 planetary satellites in all. Saturn has 6 such moons while Uranus - 5, Jupiter -4 , Neptune -1 , the Earth -1 , and Pluto -1 .

Because of the elasticity of composite materials such moons could get nearly spherical shape, and their level surface would coincide with their actual surface. However, that is not the case in fact, which indicates that actual conditions deviate from the hydrostatic equilibrium.

\section{References}

Byalko A.V.: 1989, Earth is our planet. Moscow, Science, 240 p. (in Russian).

Konstantinovskaya L.V.: 2002, Math. Meth. Geol. Cycl., 9. Konstantinovskaya L.V.: 2008, Math. Meth. Geol. Cycl., 14.

Natural satellites of the planets: [http://nfm1.sai.msu.ru/ neb/rw/natsat].

Ruskol E.L.: 2002, The Earth and the Universe, 2.

Vidmachenko A.P., Morozhenko O.V.: 2014, The physical characteristics of surfaces Earth-like planets, dwarf planets and small planet and their satellites from distance research Kyiv, Prophi (in Ukrainian). 